OPEN ACCESS

Edited by:

Jianfeng Dai,

Soochow University, China

Reviewed by:

Sumio Ohtsuki,

Kumamoto University, Japan

Weihuan Fang,

Zhejiang University, China

*Correspondence:

Ruiping She

sheruiping@126.com

tThese authors have contributed equally to this work

Specialty section:

This article was submitted to Virus and Host,

a section of the journal

Frontiers in Cellular and Infection Microbiology

Received: 15 November 2018 Accepted: 19 February 2019

Published: 19 March 2019

Citation:

Tian J, Shi R, Liu T, She R, Wu Q, An J, Hao W and Soomro MH (2019)

Brain Infection by Hepatitis $E$ Virus

Probably via Damage of the Blood-Brain Barrier Due to Alterations of Tight Junction Proteins.

Front. Cell. Infect. Microbiol. 9:52. doi: 10.3389/fcimb.2019.00052

\section{Brain Infection by Hepatitis E Virus Probably via Damage of the Blood-Brain Barrier Due to Alterations of Tight Junction Proteins}

\author{
Jijing Tian ${ }^{1+}$, Ruihan Shi ${ }^{2+}$, Tianlong Liu ${ }^{1}$, Ruiping She ${ }^{1 *}$, Qiaoxing $\mathrm{Wu}^{1}$, Junqing $A{ }^{1}$, \\ Wenzhuo Hao ${ }^{1}$ and Majid Hussain Soomro ${ }^{1}$ \\ ${ }^{1}$ Laboratory of Animal Pathology and Public Health, Key Laboratory of Zoonosis of Ministry of Agriculture, College of \\ Veterinary Medicine, China Agricultural University, Beijing, China, ${ }^{2}$ Institute of Animal Husbandry and Veterinary Medicine, \\ Beijing Academy of Agriculture and Forestry Sciences, Beijing, China
}

Extrahepatic injury, particularly neurologic dysfunctions such as Guillain-Barré syndrome, neurologic amyotrophy, and encephalitis/meningoencephalitis/myositis were associated with HEV infection, which was supported by both clinical and laboratory studies. Thus, it is crucial to figure out how the virus invades into the central nervous system (CNS). In this study, CNS lesions were determined in rabbits and Mongolian gerbils inoculated with genotype $4 \mathrm{HEV}$. Junctional proteins were detected in HEV infected primary human brain microvascular cells (HBMVCs). Viral encephalitis associated perivascular cuffs of lymphocytes and microglial nodules were observed in HEV infected rabbits. Both positive- and negative-strand of HEV RNA was detected in brain and spinal cord in rabbits intraperitoneally infected with HEV at 28 dpi (days postinoculation), but not in rabbits gavaged with HEV. HEV ORF2 protein was further examined in both brain and spinal cord sections of infected rabbits, with positive signals located mainly in neural cells and perivascular areas. Ultrastructural study showed thickened and reduplicated basement membranes of capillary endothelium in HEV RNA positive brain tissues. In vitro study showed loss of tight junction proteins including Claudin5, Occludin, and ZO-1 (zonula occludens-1) in HBMVCs inoculated with HEV for $48 \mathrm{~h}$. These findings indicated that disruption of the blood-brain barrier (BBB) might be potential mechanisms of HEV invasion into the CNS. It provides new insights to further study HEV associated neurologic disorders and will be helpful for seeking potential therapeutics for HEV infection in the future.

Keywords: Hepatitis E virus, pathological changes, blood-brain barrier, tight junction, human brain microvascular endothelial cells

\section{INTRODUCTION}

Hepatitis E virus (HEV) infection is a global public health problem. HEV is the leading cause of acute hepatitis in the world, which is responsible for 20 million infections and 70,000 HEV-related deaths annually (Montpellier et al., 2018). There are 4 genotypes of HEV, while only genotype 3 and 4 which are zoonotic, were reported in both domestic animals such as swine and human beings. We have reported infection of HEV in Himalayan Griffons foodborne, suggesting a interspecies 
transmission of HEV (Li H. et al., 2015). Foodborne transmission of HEV between animals and human through preparing or eating animal products was also reported in many countries (Tei et al., 2003; Li et al., 2005; Renou et al., 2014b). HEV can also be transmitted by blood donation or liver transplantation (Dalton and Izopet, 2018). Moreover, Watercourses contaminated by HEV-infected animals or animal products, either wild or domestic animals involved in agricultural practices, can be potential sources of infectious HEV (Doceul et al., 2016; Li et al., 2017). A recent study showed that contacting with pets and farm animals resulted in a high frequency of $\mathrm{HEV}$ infection (Abravanel et al., 2018). Thus, food and water contamination by HEV as well as directly contacting with HEV infected animals might be the most potential risks of $\mathrm{HEV}$ infection between animals and human beings.

To date, multiple experimental models of HEV were established, including animal models and cell culture systems. Animals, especially non-human primate species including chimpanzee, owl monkey, squirrel monkeys, and rhesus macaques were proved to be susceptible to HEV (Vitral et al., 1998). HEV infection in swine and other laboratory animals such as rabbits and Mongolian Gerbils provided useful tools in further understanding HEV-associated injuries (Li et al., 2009; Mao et al., 2014; Yang et al., 2015; Dalton and Izopet, 2018). Recent studies showed that several cell lines or primary cells can be used for modeling HEV infection, including human liver cell lines PLC.PRF/5, Huh-7, and HepG2/C3A, human lung epithelial cell line A549, mouse embryonic fibroblasts, and various human neural cells lines induced pluripotent stem cell-derived human neurons and primary mouse neurons (Zhang and Wang, 2016; Zhou et al., 2017). Both laboratory animals and cell cultural systems are helpful to further explain mechanisms underlying HEV infections, to develop vaccines and therapeutics for the prevention and treatment of $\mathrm{HEV}$ infections.

In recent years, extrahepatic injury of $\mathrm{HEV}$, including renal diseases, reproductive system disorders, as well as pancreatitis, and a variety of neurological disorders after HEV infection, were documented (Soomro et al., 2016, 2017; Dalton et al., 2017; Pischke et al., 2017). Neurological syndromes of HEV infection, including neuralgic amyotrophy, cerebral ischemia or infarction, seizure, encephalitis and acute combined facial, and vestibular neuropathy, were observed in a clinical cohort study in patients with non-traumatic neurological illnesses (Dalton et al., 2017). A clinical cohort study in France showed that 137 out of $200 \mathrm{HEV}$-infected patients (16.5\%) suffered from neurological manifestations (Abravanel et al., 2018), highlighting that the central nervous system (CNS) are targets of $\mathrm{HEV}$ infection. Another study showed that HEV RNA and viral protein ORF2 could be detected in brain tissues of mice and monkeys infected with HEV experimentally (Zhou et al., 2017). We have shown that various pathological changes of the CNS in Mongolian gerbils were correlated with $\mathrm{HEV}$ infection, such as perineural invasion, neuron necrosis, microglia nodule, lymphocyte infiltration, perivascular cuff and myelin degeneration. The blood-brain barrier (BBB) associated proteins such as ZO-1 (zonula occludens-1) and GFAP (glial fibrillary acidic protein) were further confirmed dysfunction in $\mathrm{HEV}$ infected animals (Shi et al., 2016). However, how the virus transmitted into the CNS and lead tissue damages are still not clear. The aim of the present study was to investigate pathological changes of the CNS in rabbits experimentally infected with $\mathrm{HEV}$ and roles of junctional complex proteins such as Claudin5, Occludin, ZO-1, and VE-cadherin in primary human brain microvascular endothelial cells (HBMVECs) during HEV infection.

\section{MATERIALS AND METHODS}

\section{Virus, Inoculation, Animals, and Sampling}

The HEV strain used for inoculation was genotype 4 swine HEV, which was derived from a swine intestinal content (GenBank accession number KJ123761) (Wu et al., 2017). Briefly, infectious virus stock was generated from the fecal samples of 2 rabbits inoculated with the intestinal content suspension intraperitoneally, with a titer of $6.63 \times 10^{7}$ copies per $\mathrm{ml}$ and stored at $-80^{\circ} \mathrm{C}$ prior to inoculation.

Thirty two 80-day-old female New Zealand white rabbits weighing between 1,800 and 2,000 $\mathrm{g}$ were purchased from the Xing Long Experimental Animal Center, Beijing, China. The blood, feces and serum of all the rabbits were confirmed to be negative for HEV RNA or HEV antigen and antibodies before inoculation. Each rabbit in the experimental groups was inoculated with $10 \mathrm{~mL}$ of prepared viral homogenate per day via intraperitoneal injection or gavage for 7 consecutive days. Rabbits inoculated with the same dose of HEV-negative intestinal homogenate served as control group. Each rabbit was housed in a separate cage, and monitored every day. No clinical symptoms were observed in the experimental rabbits. The detailed protocol was described as Wu et al. (2017).

Rabbits were sacrificed at 7,28 , and 49 days post-inoculation (dpi) of the intraperitoneal injection group (labeled with $7 \mathrm{~A}-7 \mathrm{~B}$, $28 \mathrm{~A}-28 \mathrm{D}, 49 \mathrm{~A}-49 \mathrm{D})$, and 49 and $63 \mathrm{dpi}$ of the gavaged rabbits (labeled with 49A-C, 63A-C), respectively. Brain and spinal cord of the rabbits were collected and fixed in $2.5 \%(\mathrm{v} / \mathrm{v})$ glutaraldehyde-polyoxymethylene solution, or stored at $-80^{\circ} \mathrm{C}$ immediately for viral RNA extraction. All animal protocols were approved by the Animal Care and Use Committee of China Agricultural University and were performed humanely for alleviation of suffering.

The HEV strain used for Mongolian gerbils inoculation was genotype 4 swine $\mathrm{HEV}$, derived from a swine liver sample (CHNHB-HD-L2, GenBank accession number KM024042). Briefly, animals were inoculated with the HEV virus and sacrificed at $0,7,14,21,28,42$, and 56 days dpi. Brain and spinal cord tissues were collected and fixed in $2.5 \%(\mathrm{v} / \mathrm{v})$ glutaraldehydepolyoxymethylene solution for further examination. The detailed information was described as Shi et al. (2016).

\section{RNA Extraction and PCR Protocols}

Total RNA was extracted from brain and spinal cord tissues with the Ultrapure RNA kit (CWBIO, Beijing, China) and reverse transcription were performed using HiFi-MMLV kit (CWBIO, Beijing, China) according to the instructions of the manufacturer. RTnPCR-positive brain and spinal cord tissues were arranged for 
real-time PCR to detect the viral load. The detailed protocol was described as Wu et al. (2017).

\section{Histopathological Examinations}

The tissue samples were dehydrated and embedded in paraffin wax, and serial paraffin sections $(4 \mu \mathrm{m})$ were obtained. Shortly, the sections were immersed in three consecutive 5 min xylene washes to remove paraffin and were subsequently hydrated with five consecutive ethanol washes in descending order of concentration: 100, 95, 80,70\%, and deionized water. The paraffin sections were then stained with hematoxylin-eosin $(\mathrm{H} \& \mathrm{E})$, and histopathological changes were visualized using a light microscope (LM, BX51, Olympus Co., Japan).

\section{Immunohistochemical Staining}

Tissue sections of brain and spinal cord were prepared as described above. Immunohistochemical staining was performed using a HistostainTM-Plus kit following the manufacturer's instruction (ZSGB-BIO, Beijing, China) and then applied with 3,3'-Diaminobenzidine tetrahydrochloride (DAB, ZSGB-BIO, Beijing, China) to visualize the antigen-antibody compound, and counterstained with haematoxylin. The slides were visualized using a light microscope (LM, BX51, Olympus Co., Japan).

\section{Transmission Electron Microscope (TEM)}

Brain and spinal cord samples were cut into pieces and fixed in $2.5 \%(\mathrm{v} / \mathrm{v})$ glutaraldehyde-polyoxymethylene solution overnight at $4{ }^{\circ} \mathrm{C}$. The tissues were postfixed in $2 \%$ osmium tetroxide for $1 \mathrm{~h}$ at $4^{\circ} \mathrm{C}$ and embedded in araldite CY212 after dehydration in ascending grades of ethanol. Ultrathin sections $(50-60 \mathrm{~nm})$ were sliced and stained with alkaline and lead citrate uranyl acetate. The sections were examined under a JEM 1230 transmission electron microscope.

\section{Cell Culture}

Primary human brain microvascular endothelial cells (HBMVECs) (BK-PM-010) were purchased from a company (Biopike Technology Company Ltd., Beijing, China) and cultured as described previously (Renou et al., 2014a). Briefly, cells were inoculated with HEV (300 multiplicity of infection) and collected in $48 \mathrm{~h}$ for immunofluorescence staining or western blotting. HEV-negative homogenate served as control. The data shown in immunofluorescence staining images were from one typical experiment out of three independent experiments and western blot below represented at least 3 experiments with similar results.

\section{Immunofluorescence Staining}

Cells were fixed in $4 \%$ formaldehyde and permeabilized in $0.2 \%$ Triton X-100/PBS. After washing and blocking with $2 \%$ bovine serum albumin, the sections were incubated with antibodies for laudin5 (1:200; Boster Biological Technology Co., Ltd., China) or Vementin (1:200; Beijing Bioss Biological Technology Co., Ltd., China) overnight at $4^{\circ} \mathrm{C}$. After washing, the appropriate secondary antibodies were applied (1:2000; CoWin Biosciences, China) and the nucleus were stained with DAPI. The images were analyzed by Fluorescence microscopy.

\section{Western Blotting}

The protein concentration from HEV-treated human cerebral microvascular endothelial cells was determined with the BCA protein assay kit (Thermo Fisher Scientific, Waltham, MA, USA) and $30 \mu \mathrm{g}$ of protein was separated by SDS-PAGE. The blots were incubated with anti-Claudin5 (1:600) and ZO1 (1:500) purchased from Boster Biological Technology Co., Ltd. (China), Occludin (1:500) and VE-cadherin (1:500) from Beijing Bioss Biological Technology Co., Ltd. (China), and anti$\beta$-tubulin (1:600; Boster Biological Technology Co., Ltd., Wuhan, China) as internal control for $16 \mathrm{~h}$ at $4^{\circ} \mathrm{C}$. The membranes were then incubated with the secondary antibodies conjugated with horseradish peroxidase (Santa Cruz Biotechnology) and developed by enhanced chemiluminescence (Thermo Scientific). The images were analyzed with ImageJ (NIH, Bethesda, MD, USA) and data were normalized to the expression of anti- $\beta$ tubulin antibody.

\section{Statistical Analysis}

The data shown represent at least 3 experiments and were analyzed by the 2-tailed, unpaired Student's $t$-test between 2 groups. The results are expressed as the mean \pm SEM. $P<0.05$ was considered statistically significant.

\section{RESULTS}

\section{Evaluation of Positive- and Negative-Strand of HEV RNA in Brain and Spinal Cord}

To evaluate the virus infection and replication, HEV RNA in brain and spinal cord tissue of infected rabbits was detected by RT-nPCR at 7, 28, 48 dpi after intraperitoneal injection and 49 dpi, 63 dpi after gavage, and results were summarized in Table 1. The results showed that all the brain and spinal cord from control rabbits were tested negative for HEV RNA. Two rabbits infected via intraperitoneal injection were detected HEV RNA positive in the brains at $28 \mathrm{dpi}$, with viral load $2.90 \mathrm{E} 2$ and $1.18 \mathrm{E} 2$ copies $/ \mu \mathrm{l}$, respectively. One rabbit was detected HEV RNA positive in spinal cord at $28 \mathrm{dpi}$ in intraperitoneal injection group, with viral load

TABLE 1 | Viral load detection in brain and spinal cord of experimentally infected rabbits.

\begin{tabular}{|c|c|c|c|c|c|}
\hline \multirow[t]{2}{*}{ Sample/dpi } & \multicolumn{3}{|c|}{ Via intraperitoneal injection } & \multicolumn{2}{|c|}{ Via gavage } \\
\hline & $7 \mathrm{dpi}$ & $28 \mathrm{dpi}$ & $49 \mathrm{dpi}$ & $49 \mathrm{dpi}$ & $63 \mathrm{dpi}$ \\
\hline HEV-positive brain No. & - & $28 C, 28 D^{a}$ & - & - & - \\
\hline Viral load in brain & - & 2.90E2, 1.18E2 & - & - & - \\
\hline HEV-positive spinal cord No. & - & $28 C^{b}$ & - & - & - \\
\hline Viral load in spinal cord & - & 4.35E2 & - & - & - \\
\hline
\end{tabular}

DPI, days postinoculation. -, positive-strand and negative-strand of HEV RNA negative by RT-nPCR.

a brain positive-strand and negative-strand HEV RNA-positive rabbit serial number by $R T-n P C R$.

${ }^{b}$ spinal cord positive-strand and negative-strand HEV RNA-positive rabbit serial number by RT-nPCR.

HEV viral load in brain and spinal cord are detected by qPCR and shown as copies/ $\mu$ l. 


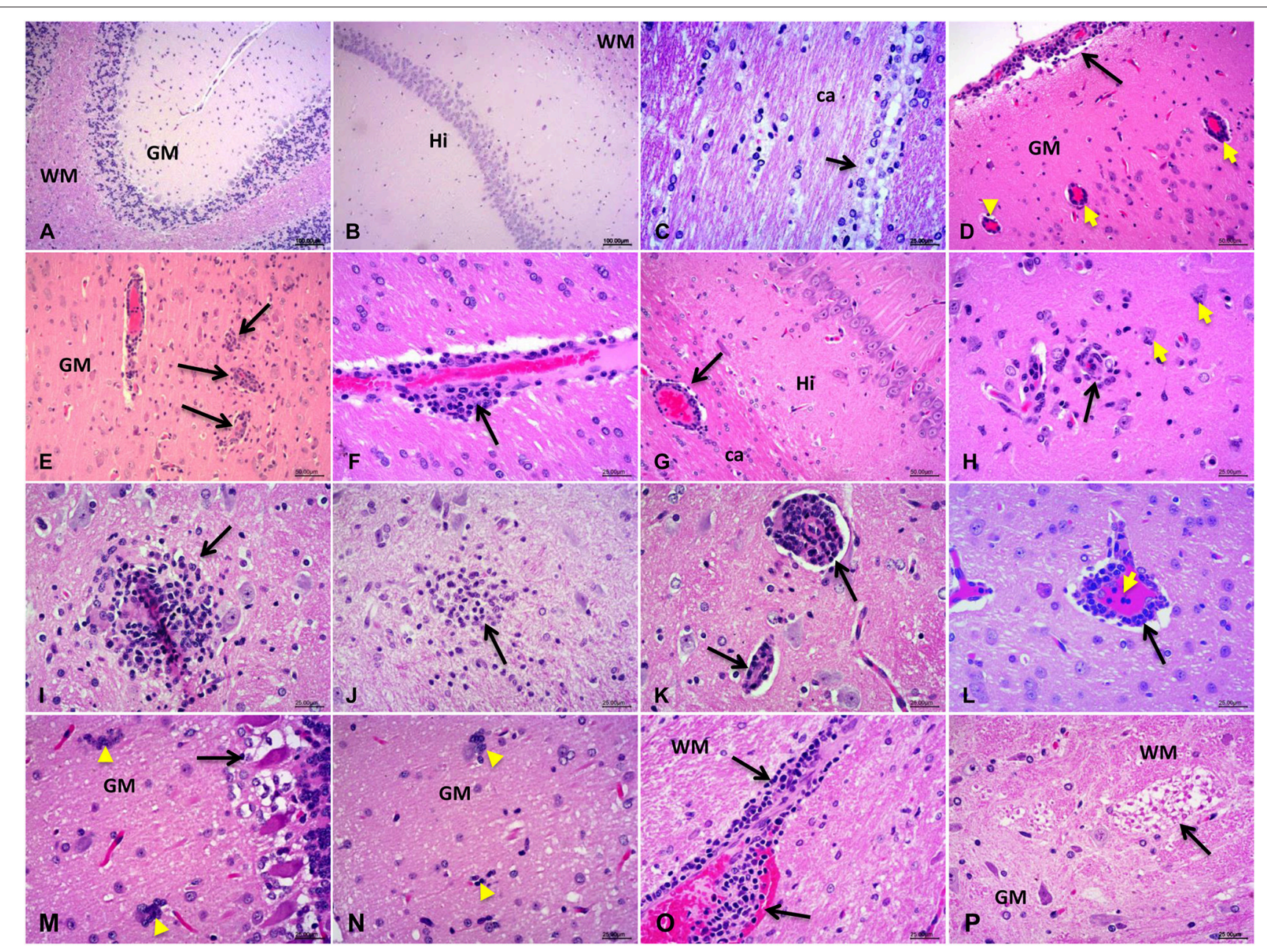

FIGURE 1 | Histopathological detection of the central nervous system (CNS). (A-C) The normal structure of the CNS tissue from control rabbits: cerebellum (A) the molecular layer and granular layers of the gray matter (GM), and pukinje cells and the white matter (WM); cerebrum (B) hippocampus (Hi) and (C) corpus callosum (ca) and the ependymal epithelium of the white matter (D-P). Various pathological changes observed in HEV-RNA positive brain sections, especially the characteristics of viral encephalitis include perivascular cuffs of lymphocytes and microglial nodules: cerebrum (D-I): venous congestion of the Pia Mater, perivascular cuffs of lymphocytes and microglial hyperplasia in the cerebral cortex, corpus callosum (ca), brainstem, hypothalamus and the pons; cerebellum (M) degenerate and necrotic pukinje cells, (N) microglial nodules, and necrotic neurons surrounded and invaded by hypertrophic microglial cells (neuronophagia), (O) perivascular cuffs of lymphocytes, and hemorrhage; spinal cord $\mathbf{( P )}$ : degeneration of neuron axons of the white matter (demyelination).

4.35E2 copies/ $\mu$ l. Rabbits infected HEV via gavage were not tested positive at 49 and $63 \mathrm{dpi}$.

\section{Histopathological Lesions of Brain and Spinal Cord of Rabbits Experimentally Infected by HEV}

The results obtained by $\mathrm{H} \& \mathrm{E}$ staining showed typical viral encephalitis changes in HEV-RNA positive rabbits, including meningoencephalitis, perivascular cuffs of lymphocytes and microglial nodules in the cerebrum and cerebellum tissues in rabbits experimentally infected by HEV (Figures 1D-L). Degradation and necrosis of neurons and Pukinje cells were found in the cerebellum sections of the infected rabbits (Figures 1M,N). Neuron axons degeneration (demyelination) of the white matter in spinal cord of experimental rabbits was examined (Figure 1P). Additionally, vascular congestion and hemorrhage occurred commonly in brain and spinal cord of the experimental rabbits. No obvious injuries were observed in control rabbits (Figures 1A-C).

\section{Immunohistochemistry Staining for HEV ORF2 Antigen in Brain and Spinal Cord}

To figure out the expression of HEV antigen in brain and spinal cords, IHC staining was conducted for HEV ORF2 protein. ORF2 antigen was detected in brain and spinal cord in rabbits detected as HEV-positive. Positive signals distributed mainly in cytoplasm of neuroglial cells and choroid epithelium cells in cerebrum (Figures 2D-G); and in pia mater and injured neurons, Pukinje cells in cerebellum (Figures $\mathbf{2 H}, \mathbf{I}$ ); and in neurons of the pons (Figure 2J). In the spinal cord, HEV ORF2 protein was visible 


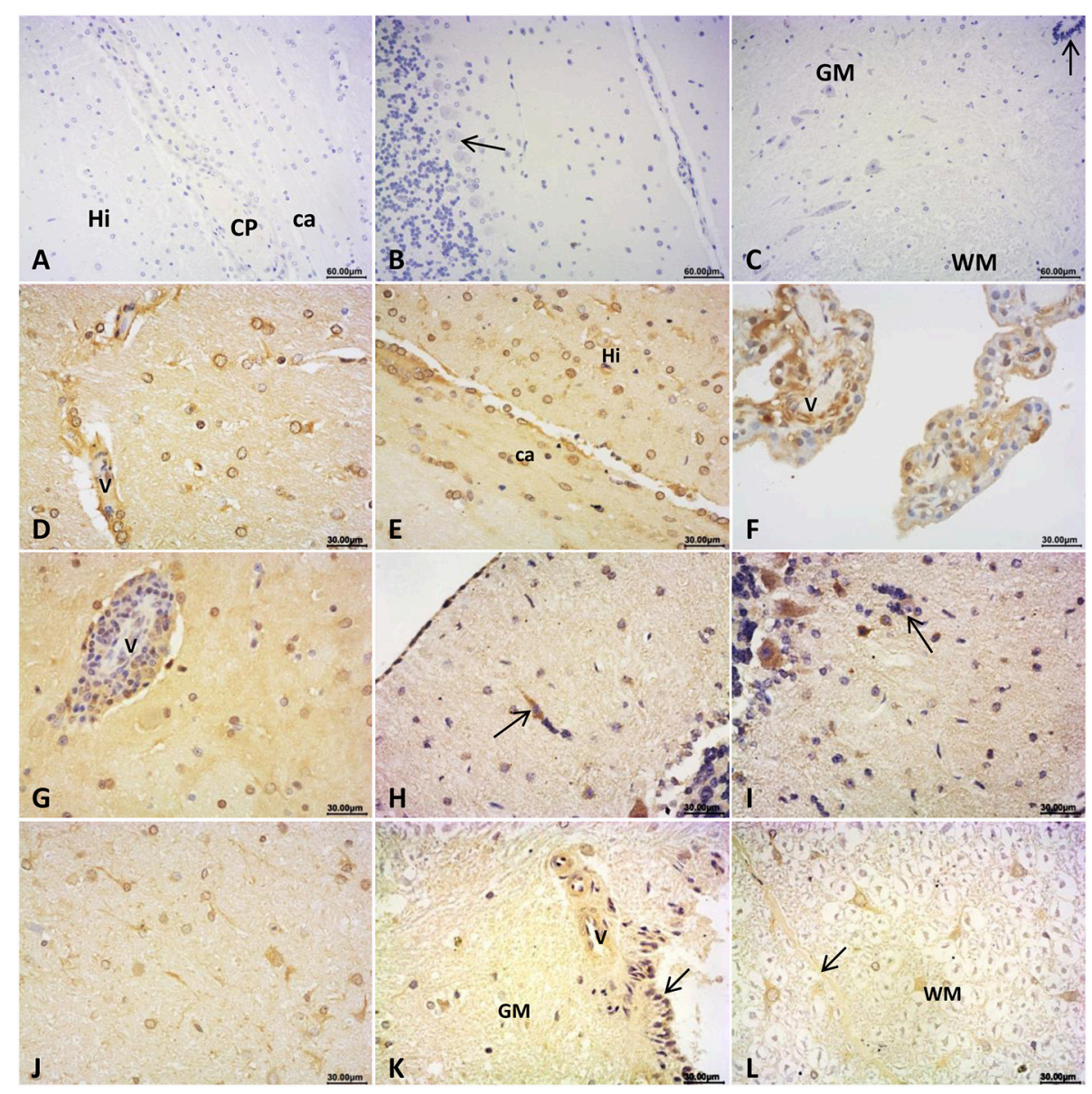

FIGURE 2 | Immunohistochemical staining for HEV ORF2 antigen. (A-C) ORF2 positive signals was not detected in brain and spinal cord of the control rabbits: cerebrum (A) hippocampus and white matter area (Hi: hippocampus, CP: choroid plexus, ca: corpus callosum), cerebellum (B) the molecular layer and granular layers, and pukinje cells, spinal cord (C) the gray matter (GM) and white matter (WM), arrow showed central canal of the spinal cord. In brain detected as HEV RNA positive, HEV ORF2 protein expression distributed at: (D-G) cerebrum, (D) perivascular area and cytoplasm of glial cells of the white gray, (E) cytoplasm of glial cells in corpus callosum (ca) and hippocampus (Hi) of the white matter, (F) cytoplasm of choroid epithelium cells, and (G) lymphocytes in perivascular area and glial cells cytoplasm of the gray matter; (H-I) cerebellum, $\mathbf{( H )}$ Pia Mater and neurons invaded by microglial cells, (I) cytoplasm of pukinje cells and cells that formed microglial nodules (arrow); (J) cytoplasm of neurons and glial cells in white matter of pons; (K,L) spinal cord, (K) perivascular area and epithelial cells of central canal of the gray matter, (L) posterior septum (arrow) of spinal cord and cytoplasm of neurons in white matter (WM).

in cytoplasm of neurons (Figures $2 \mathrm{~K}, \mathbf{L}$ ). No positive signal was observed in the CNS from control rabbits (Figures 2A-C).

\section{Ultrastructure of BBB Injury After HEV Infection}

Transmission electron microscope (TEM) was used to determine morphological injury of $\mathrm{BBB}$. No apparent lesions were observed in brain and spinal cord tissues of control gerbils. Basement membranes of capillary endothelium were smooth and continuous with uniform thickness and junctional complexes between the endothelium were compact and clearly defined (Figures 3A-C). In HEV RNA-positive tissues from the experiment group, disruption of $\mathrm{BBB}$ was determined, including irregular and thickened basement membranes and indistinct junctional complexes between endothelial cells of spinal cord tissues (Figures 3E,F). In brain tissues with HEV RNA, it was found that basement membranes of endothelium and surrounded pericytes were thickened and reduplicated (Figure 3D).

\section{Tight Junction Related Proteins Claudin5, Occludin, and ZO-1 Decreased in HBMVECs Experimentally Infected With HEV}

To further explore expression of tight junction proteins during HEV infection, Claudin5, Occludin, and ZO-1 were examined by both immunofluorescence staining and western blot in HBMVECs infected with HEV. Under the microscopy, expression of Claudin 5 in HBMVECs were consecutively located in membrane of the cells in control group; Claudin5 positive signals in HEV-infected cells decreased and aggregated in cell cytoplasm (Figure 4A). Western blot showed expression levels of Claudin 5 were significantly decreased in experimental 


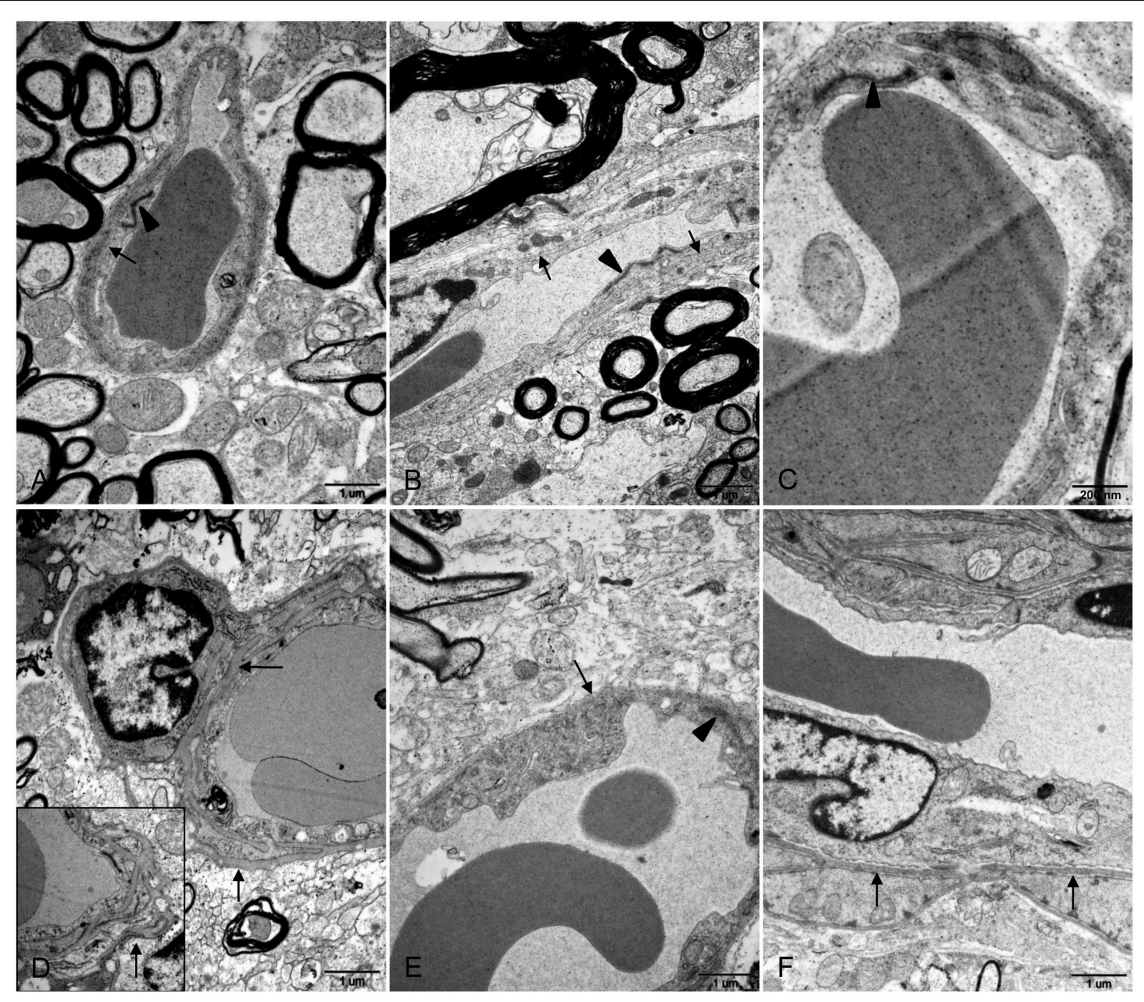

FIGURE 3 | Ultrastructural examination of BBB in brain and spinal cord tissues. (A-C) Basement membranes of blood vessel wall was intact and smooth (arrows) and junctional complexes between the endothelium were compact and clearly defined (arrowheads) in control brain (A) and spinal cord (B,C) tissues. (D-F) In brain tissues from HEV RNA-positive gerbils, basement membranes of capillary endothelium as well as pericytes was thickened and reduplicated (arrows) (D). In spinal cord tissues positive with HEV RNA, basement membranes of blood vessel endothelium was irregular and thickened (arrows) (E,F), and junctional complexes between endothelial cells were indistinct (arrowhead) (E).

cells compared to control $(P<0.05)$ (Figure 4B). Levels of Occludin and ZO-1 were also reduced in HEV-treated cells $(P<$ 0.05) (Figures 4C,D).

\section{Adhesion Junction Related Protein VE-Cadherin Increased in HEV Infected HBMVECs}

To further investigate changes of adhesion junction protein during HEV infection, VE-cadherin were tested in HBMVECs infected with HEV. Under the microscopy, VE-cadherin expressed as scattering points along cell membrane between two adjacent cells in control group; expression levels of VEcadherin increased in HEV-infected cells compared to control group (Figure 5A). Western blot showed increased protein expression of VE-cadherin in HEV-infected HBMVECs $(P<$ 0.05) (Figure 5B).

\section{Expression of Vimentin Did Not Change Significantly in HEV Infected HBMVECs}

To determine whether HEV infection alters cell stability, a cytoskeleton protein Vimentin was examined in HBMVECs infected with HEV. Western blot showed no significant change of Vimentin expression in cells infected with HEV (Figure 6).

\section{DISCUSSION}

A number of studies have reported neurological manifestations in HEV-infected patients or laboratory animals, with evidence of HEV replication in neuronal cells (Pischke et al., 2017). It was found that Guillain-Barré syndrome with abnormal alanine aminotransferase (ALT) levels usually associated with HEV infection (Stevens et al., 2017). Our previous data showed that ALT level was significantly increased in the experimentally infected rabbits at 28 and $49 \mathrm{dpi}$, and HEV RNA was detected in multiple tissues, including the liver, salivary gland, tonsil, spleen, lymph nodes, small intestine, Sacculus Rotundus (SR), cecum, and appendices (Wu et al., 2017). In the present study, HEV RNA (positive and negative strand) was tested in brain (2/4) and spinal cord (1/4) at $28 \mathrm{dpi}$ in experimental rabbits inoculated with $\mathrm{HEV}$ via intraperitoneal injection. These results further confirm that rabbits is susceptible to HEV under experimental conditions, with a risk of $\mathrm{CNS}$ infection.

To date, very limited pathological injuries were documented in clinical and laboratory studies of HEV infection, though a number of studies have reported that $\mathrm{HEV}$ infection associated with clinical syndromes such as Guillain-Barré syndrome, neuralgic amyotrophy, and encephalitis/meningoencephalitis/myositis (Dalton et al., 

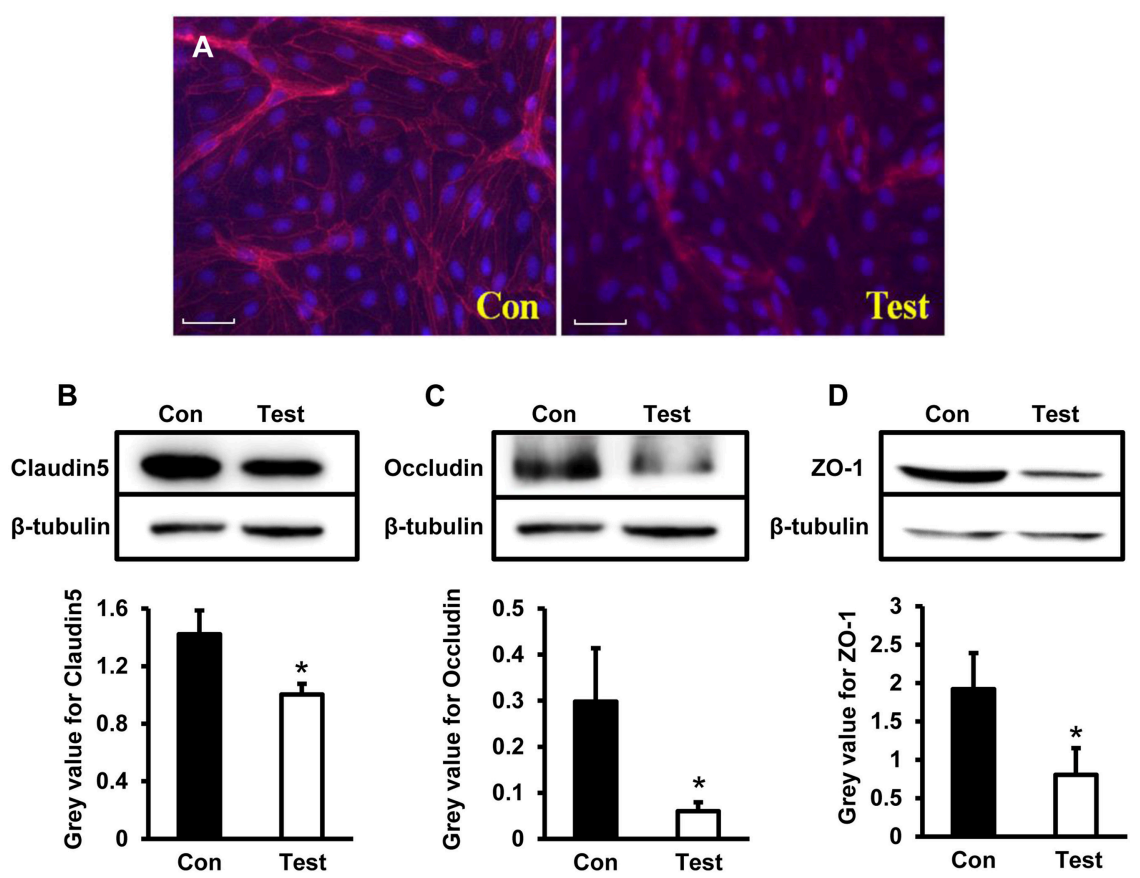

FIGURE 4 | Quantity analysis of tight junction-associated proteins (Claudin5, Occludin, ZO-1) in HBMVECs. (A) Representative images of Immunofluorescence staining. The images showed that positive signals of Claudin5 distributed consecutively in membrane of control cells, and expression levels decreased after HEV infection and positive signals translocated into cell cytoplasm (Red: Claudin, Blue: DAPI). (B) Western blot indicated that expression levels of Claudin5 were significantly reduced in HEV-infected cells compared to control group $\left.{ }^{\star} P<0.05\right)$. (C,D) Deceased protein levels of Occludin and ZO-1 were also detected in HEV-infected cells $\left({ }^{\star} P<0.05\right)$.
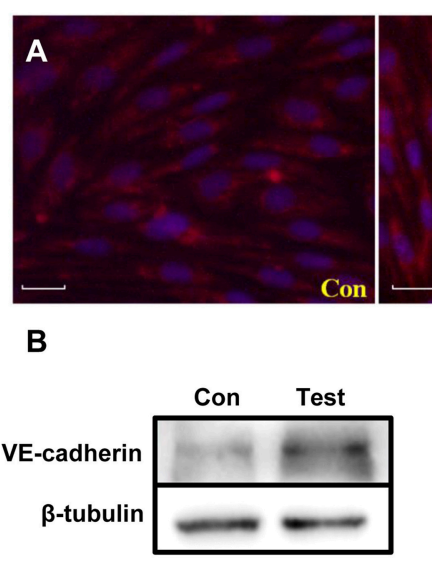

B
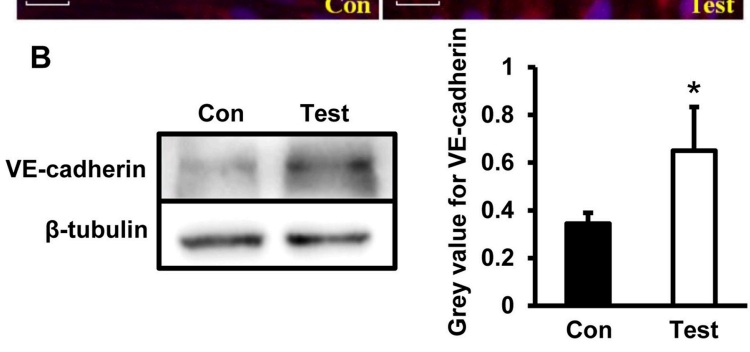

FIGURE 5 | Quantity analysis of adherent junction protein (VE-cadherin) in HBMVECs. (A) Representative images of Immunofluorescence staining. The images showed that VE-cadherin expressed as scattering points around cell membrane between two adjacent cells in control cells, and positive signals increased in HEV-infected cells (Red: VE-cadherin, Blue: DAPI). (B) Western blot analysis showed that VE-cadherin expression levels were increased in HEV-infected HBMVECs $\left({ }^{\star} P<0.05\right)$.

2016). In gerbils experimentally infected HEV, pathological changes, including neurons degeneration and necrosis,

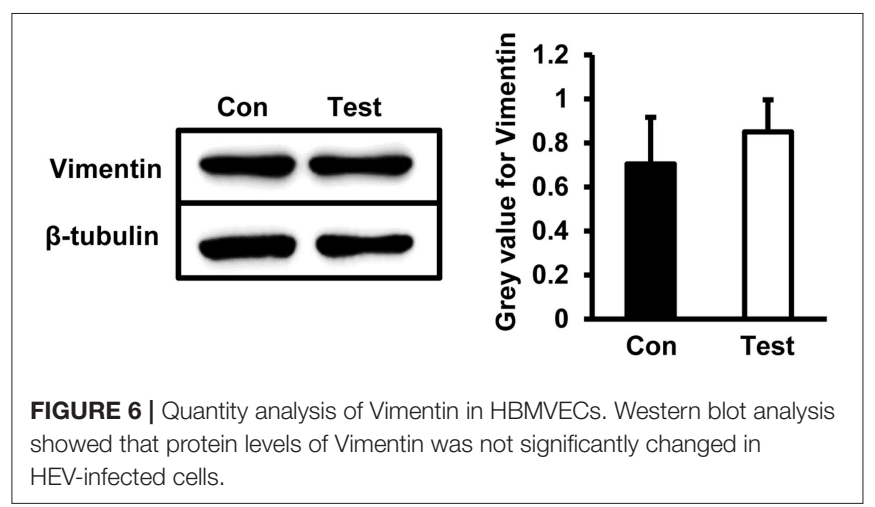

neuronophagia, microglia nodules, inflammatory cells infiltrating to the ependymal epithelium, choroid plexus region hemorrhage and inflammatory cells infiltration, "perivascular cuff" formation, Purkinje cells necrosis, hemorrhage in dorsal median septum and central canal of spinal cord were observed (Shi et al., 2016). These pathological changes were in accordance with clinical manifestations in HEV infected patients. Rabbits are considered to be useful model to explore the pathogenesis of hepatitis E, especially the extrahepatic injury of HEV (Nimgaonkar et al., 2018). In our study, pathological changes particularly viral encephalitis associated perivascular cuffs of lymphocytes and microglial nodules were observed in rabbits infected with HEV. Together with neuron injuries 
detected, our results indicated that HEV might directly involve in the CNS disorders.

There are 3 opening reading frames (ORF) of $\mathrm{HEV}$, including the viral replicase (belongs to ORF1), the viral capsid protein (ORF2), and a small phosphoprotein might involve in the secretion of viral particles (ORF3) (Debing et al., 2016). However, a recent study showed that only ORF2 was unequivocally detectable in FFPE liver samples with hepatitis E, and the distribution pattern of HEV ORF2 particles was not only in cytoplasm and bile canaliculi but also in nucleus of hepatocytes (Lenggenhager et al., 2017). Most importantly, they found that HEV ORF2 expression correlated with the detection of HEV RNA in situ by ISC, and IHC for ORF2 protein, indicating that ORF2 was sufficiently sensitive for HEV detection in a period of high viral load (Lenggenhager et al., 2017). Interestingly, HEV ORF2 protein was observed in the cerebellum granule cell layer in brain tissue of infected mice and chronically infected monkeys (Zhou et al., 2017). In vitro experiments further revealed that both primary cerebellar and hippocampal neurons of mice were susceptible to HEV infection and capable of producing the viral ORF2 protein (Zhou et al., 2017). In our study, HEV ORF2 particles were detected in cytoplasm or nucleus of cells in brain and spinal cord tissues of the HEV RNA positive rabbits, such as glial cells, microglial cells, choroid epithelium cells, and neural cells, especially in cells located in perivascular areas. These results suggested that perivascular cells and neural cells are targets of HEV in CNS, which is in accordance with our previous study in gerbils (Shi et al., 2016). In gerbils experimentally infected with $\mathrm{HEV}$, neurocytes degeneration and necrosis, particularly the blood vessel wall components of the brain and spinal cord sections, including swelling endothelium and loss of endothelial junctional complexes, were observed using a transmission electron microscopy (Shi et al., 2016).
We also found that basement membranes of the blood vessel was thickened and even reduplicated in brain and spinal cord tissues of HEV infected gerbils, which might be a compensatory response to $\mathrm{BBB}$ disruption. Together, these findings suggested that $\mathrm{HEV}$ infection result in a leakage of the $\mathrm{BBB}$, which facilitated the virus transmission into the central nervous tissue.

The BBB is comprised of highly specialized and tightly sealed monolayer of brain microvascular endothelial cells (BMVECs), and other immune and neural cells, with elaborate junctional complex (both tight junctions and adherens junctions such as ZO-1, Claudins, Occludin, VE-cadherin) and integrated basal lamina which restricted the entry of pathogens and other soluble molecules into the CNS (Bernas et al., 2010; Miner and Diamond, 2016). Junctional proteins were reported to play critical roles during neurological disorders such as experimental autoimmune encephalomyelitis (EAE), Alzheimer's disease and Parkinson's disease (Pfeiffer et al., 2011; Cabezas et al., 2014; Halliday et al., 2016). Besides, neurotropic viruses such as West Nile virus, Japanese encephalitis virus (JEV) and Rabies virus infection are causes of $\mathrm{BBB}$ disruption which in turn facilitate the spread of virus into brain parenchyma from blood circulation. Loss of ZO-1 was detected in HBMVE cells incubated with supernatant media from WNV-infected HBCA cells (Verma et al., 2010). Occludin expression was downregulated by CXCL10 in bEnd. 3 cells co-cultured with brain extracts infected with RABV (Chai et al., 2015). Expression of tight junction proteins occludin, claudin-5 and ZO-1 in JEVinfected mice brain were overall reduced (Li F. et al., 2015). The above study indicated that directly crossing the $\mathrm{BBB}$ is one of the most common pathways of viruses invading the CNS, and breaking the junctional complexes integrity will be a key factor. In the present study, primary HBMVECs were employed to evaluate roles of tight junctions and adherens

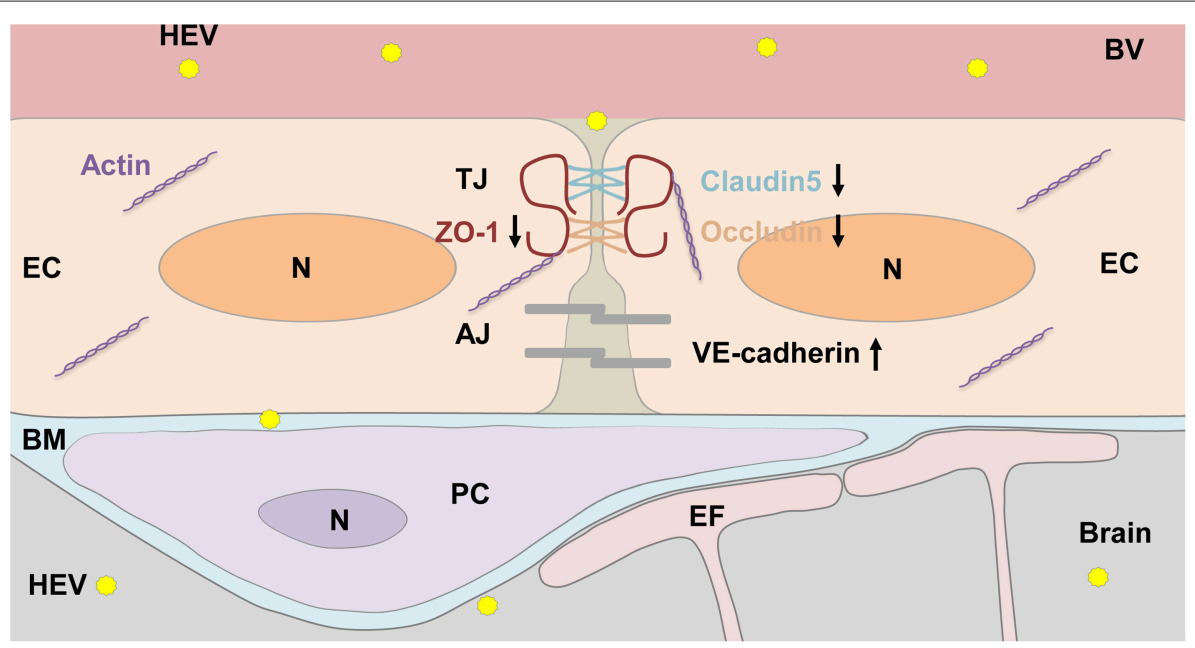

FIGURE 7 | HEV entry to the brain. Components of the BBB including tight junction (TJ) and adhesion junction (AJ) between endothelial cells (EC), pericytes (PC), astrocytes endfoot (EF), as well as basement membrane (BM) surrounded ECs and PCs were shown in the graph. HEV infection decreased expression levels of TJ proteins, including ZO-1, Occludin, and Claudin5, increased AJ protein VE-cadherin expression, leading disorder of junctional complexes between capillary ECs, further facilitating HEV invasion into the brain tissue. In responding, basement membrane of PCs and ECs became thickened to protect brain tissue from HEV infection. N, Nucleus; BV, blood vessel. 
junctions during HEV infection. We found that expression of tight junction proteins Claudin5, Occludin, and ZO-1 decreased in HBMVECs treated with HEV, indicating a direct invasion of $\mathrm{HEV}$ into the CNS via disruption of tight junction complex of HBMVECs. However, further investigation showed that VEcadherin, an adherens junction protein participating into the cohesion and organization of the intercellular junctions of endothelial cells, was upregulated in HBMVECs infected with HEV. It might be a compensatory response to dysfunction of tight junction during HEV invasion. The cytoskeleton protein Vimentin was not changed in the infected cells, indicating that HBMVECs stability might not be largely affected by HEV infection.

In summary, the $\mathrm{BBB}$ of brain is a potential target of HEV invasion into the CNS in experimentally infected rabbits (Figure 7). Further study should be focused on roles of tight junction proteins such as Claudin5, Occludin, and ZO-1 in integrity of $\mathrm{BBB}$ during $\mathrm{HEV}$ infection. It will

\section{REFERENCES}

Abravanel, F., Pique, J., Couturier, E., Nicot, F., Dimeglio, C., Lhomme, S., et al. (2018). Acute hepatitis E in French patients and neurological manifestations. $J$ Infect. 77, 220-226. doi: 10.1016/j.jinf.2018.06.007

Bernas, M. J., Cardoso, F. L., Daley, S. K., Weinand, M. E., Campos, A. R., Ferreira, A. J., et al. (2010). Establishment of primary cultures of human brain microvascular endothelial cells to provide an in vitro cellular model of the blood-brain barrier. Nat. Protoc. 5, 1265-1272. doi: 10.1038/nprot.2010.76

Cabezas, R., Avila, M., Gonzalez, J., El-Bacha, R. S., Baez, E., Garcia-Segura, L. M., et al. (2014). Astrocytic modulation of blood brain barrier: perspectives on Parkinson's disease. Front. Cell Neurosci. 8:211. doi: 10.3389/fncel.2014.00211

Chai, Q., She, R., Huang, Y., and Fu, Z. F. (2015). Expression of neuronal CXCL10 induced by rabies virus infection initiates infiltration of inflammatory cells, production of chemokines and cytokines, and enhancement of blood-brain barrier permeability. J Virol. 89, 870-876. doi: 10.1128/JVI.02154-14

Dalton, H. R., and Izopet, J. (2018). Transmission and epidemiology of Hepatitis E virus genotype 3 and 4 infections. Cold Spring Harb. Perspect. Med. 8:a032144. doi: $10.1101 /$ cshperspect.a032144

Dalton, H. R., Kamar, N., van Eijk, J. J., McLean, B. N., Cintas, P., Bendall, R. P., et al. (2016). Hepatitis E virus and neurological injury. Nat. Rev. Neurol. 12, 77-85. doi: 10.1038/nrneurol.2015.234

Dalton, H. R., van Eijk, J. J. J., Cintas, P., Madden, R. G., Jones, C., Webb, G. W., et al. (2017). Hepatitis E virus infection and acute non-traumatic neurological injury: a prospective multicentre study. J. Hepatol. 67, 925-932. doi: 10.1016/j.jhep.2017.07.010

Debing, Y., Moradpour, D., Neyts, J., and Gouttenoire, J. (2016). Update on hepatitis E virology: Implications for clinical practice. J. Hepatol. 65, 200-212. doi: 10.1016/j.jhep.2016.02.045

Doceul, V., Bagdassarian, E., Demange, A., and Pavio, N. (2016). Zoonotic Hepatitis E virus: classification, animal reservoirs and transmission routes. Viruses 8:270. doi: 10.3390/v8100270

Halliday, M. R., Rege, S. V., Ma, Q., Zhao, Z., Miller, C. A., Winkler, E. A., et al. (2016). Accelerated pericyte degeneration and blood-brain barrier breakdown in apolipoprotein E4 carriers with Alzheimer's disease. J. Cereb. Blood Flow Metab. 36, 216-227. doi: 10.1038/jcbfm.2015.44

Lenggenhager, D., Gouttenoire, J., Malehmir, M., Bawohl, M., HoncharovaBiletska, H., Kreutzer, S., et al. (2017). Visualization of Hepatitis E virus RNA and proteins in the human liver. J. Hepatol. 67, 471-479. doi: 10.1016/j.jhep.2017.04.002

Li, F., Wang, Y., Yu, L., Cao, S., Wang, K., Yuan, J., et al. (2015). Viral infection of the central nervous system and neuroinflammation precede blood-brain barrier help understand pathogenesis of the CNS injury caused by $\mathrm{HEV}$ and seek potential therapeutics for HEV infection in the future.

\section{AUTHOR CONTRIBUTIONS}

JT, RShi, and RShe performed the study concept and design. RShi, TLiu, QW, and JA performed the laboratory work and data analysis. WH and MS performed the analysis and interpretation of data. JT and RShi wrote the paper. All of the authors read and approved the final article.

\section{FUNDING}

This study was supported by the National Natural Science Foundation of China (Grant No. 31472165, 31802162, 31272515) and Innovative Project of Young Teachers of China Agricultural University (Grant no. 2018QC015 and 2019TC004). disruption during japanese encephalitis virus infection. J. Virol. 89, 5602-5614. doi: 10.1128/JVI.00143-15

Li, H., Li, W., She, R., Yu, L., Wu, Q., Yang, J., et al. (2017). Hepatitis E virus genotype 4 sequences detected in sewage from treatment plants of China. Food Environ. Virol. 9, 230-233. doi: 10.1007/s12560-016-9276-y

Li, H., Zhu, R., She, R., Zhang, C., Shi, R., Li, W., et al. (2015). Case report associated with aspergillosis and Hepatitis E virus coinfection in Himalayan Griffons. Biomed. Res. Int. 2015:287315. doi: 10.1155/2015/287315

Li, T. C., Chijiwa, K., Sera, N., Ishibashi, T., Etoh, Y., Shinohara, Y., et al. (2005). Hepatitis E virus transmission from wild boar meat. Emerg. Infect. Dis. 11, 1958-1960. doi: 10.3201/eid1112.051041

Li, W., Sun, Q., She, R., Wang, D., Duan, X., Yin, J., et al. (2009). Experimental infection of Mongolian gerbils by a genotype 4 strain of swine Hepatitis E virus. J. Med. Virol. 81, 1591-1596. doi: 10.1002/jmv.21573

Mao, J., Zhao, Y., She, R., Cao, B., Xiao, P., Wu, Q., et al. (2014). Detection and localization of rabbit Hepatitis $\mathrm{E}$ virus and antigen in systemic tissues from experimentally intraperitoneally infected rabbits. PLoS ONE 9:e88607. doi: 10.1371/journal.pone.0088607

Miner, J. J., and Diamond, M. S. (2016). Mechanisms of restriction of viral neuroinvasion at the blood-brain barrier. Curr. Opin. Immunol. 38, 18-23. doi: 10.1016/j.coi.2015.10.008

Montpellier, C., Wychowski, C., Sayed, I. M., Meunier, J. C., Saliou, J. M., Ankavay, M., et al. (2018). Hepatitis E virus lifecycle and identification of 3 forms of the ORF2 capsid protein. Gastroenterology 154, 211-223 e8. doi: 10.1053/j.gastro.2017.09.020

Nimgaonkar, I., Ding, Q., Schwartz, R. E., and Ploss, A. (2018). Hepatitis E virus: advances and challenges. Nat. Rev. Gastroenterol. Hepatol. 15, 96-110. doi: 10.1038/nrgastro.2017.150

Pfeiffer, F., Schafer, J., Lyck, R., Makrides, V., Brunner, S., SchaerenWiemers, N., et al. (2011). Claudin-1 induced sealing of blood-brain barrier tight junctions ameliorates chronic experimental autoimmune encephalomyelitis. Acta Neuropathol. 122, 601-614. doi: 10.1007/ s00401-011-0883-2

Pischke, S., Hartl, J., Pas, S. D., Lohse, A. W., Jacobs, B. C., and Van der Eijk, A. A. (2017). Hepatitis E virus: Infection beyond the liver? J. Hepatol. 66, 1082-1095. doi: 10.1016/j.jhep.2016.11.016

Renou, C., Gobert, V., Locher, C., Moumen, A., Timbely, O., Savary, J., et al. (2014a). Prospective study of Hepatitis E virus infection among pregnant women in France. Virol. J. 11:68. doi: 10.1186/1743-422X-11-68

Renou, C., Roque-Afonso, A. M., and Pavio, N. (2014b). Foodborne transmission of Hepatitis E virus from raw pork liver sausage, France. Emerg. Infect. Dis. 20, 1945-1947. doi: 10.3201/eid2011.140791 
Shi, R., Soomro, M. H., She, R., Yang, Y., Wang, T., Wu, Q., et al. (2016). Evidence of Hepatitis E virus breaking through the blood-brain barrier and replicating in the central nervous system. J. Viral. Hepat. 23, 930-939. doi: 10.1111/jvh.12557

Soomro, M. H., Shi, R., She, R., Yang, Y., Hu, F., and Li, H. (2016). Antigen detection and apoptosis in Mongolian gerbil's kidney experimentally intraperitoneally infected by swine Hepatitis E virus. Virus Res. 213, 343-352. doi: 10.1016/j.virusres.2015.12.012

Soomro, M. H., Shi, R., She, R., Yang, Y., Wang, T., Wu, Q., et al. (2017). Molecular and structural changes related to Hepatitis E virus antigen and its expression in testis inducing apoptosis in Mongolian gerbil model. J. Viral. Hepat. 24, 696-707. doi: 10.1111/jvh.12690

Stevens, O., Claeys, K. G., Poesen, K., Saegeman, V., and Van Damme, P. (2017). Diagnostic challenges and clinical characteristics of Hepatitis E virus-associated Guillain-Barre syndrome. JAMA Neurol. 74, 26-33. doi: 10.1001/jamaneurol.2016.3541

Tei, S., Kitajima, N., Takahashi, K., and Mishiro, S. (2003). Zoonotic transmission of Hepatitis E virus from deer to human beings. Lancet 362, 371-373. doi: 10.1016/S0140-6736(03)14025-1

Verma, S., Kumar, M., Gurjav, U., Lum, S., and Nerurkar, V. R. (2010). Reversal of West Nile virus-induced blood-brain barrier disruption and tight junction proteins degradation by matrix metalloproteinases inhibitor. Virology 397, 130-138. doi: 10.1016/j.virol.2009.10.036

Vitral, C. L., Yoshida, C. F. T., and Gaspar, A. M. C. (1998). The use of non-human primates as animal models for the study of hepatitis viruses. Braz. J. Med. Biol. Res. 31, 1035-1048.
Wu, Q., An, J., She, R., Shi, R., Hao, W., Soomro, M., et al. (2017). Detection of genotype 4 swine Hepatitis $\mathrm{E}$ virus in systemic tissues in cross-species infected rabbits. PLOS ONE 12:e0171277. doi: 10.1371/journal.pone.017 1277

Yang, Y., Shi, R., She, R., Soomro, M. H., Mao, J., Du, F., et al. (2015). Effect of swine Hepatitis E virus on the livers of experimentally infected Mongolian gerbils by swine Hepatitis E virus. Virus Res. 208, 171-179. doi: 10.1016/j.virusres.2015.06.007

Zhang, F., and Wang, Y. (2016). HEV cell culture. Adv. Exp. Med. Biol. 948, 143-159. doi: 10.1007/978-94-024-0942-0_8

Zhou, X., Huang, F., Xu, L., Lin, Z., de Vrij, F. M. S., Ayo-Martin, A. C., et al. (2017). Hepatitis E virus infects neurons and brains. J. Infect. Dis. 215, 1197-1206. doi: 10.1093/infdis/jix079

Conflict of Interest Statement: The authors declare that the research was conducted in the absence of any commercial or financial relationships that could be construed as a potential conflict of interest.

Copyright (C) 2019 Tian, Shi, Liu, She, Wu, An, Hao and Soomro. This is an openaccess article distributed under the terms of the Creative Commons Attribution License (CC BY). The use, distribution or reproduction in other forums is permitted, provided the original author(s) and the copyright owner(s) are credited and that the original publication in this journal is cited, in accordance with accepted academic practice. No use, distribution or reproduction is permitted which does not comply with these terms. 